\title{
Análise tricológica em morcegos do Brasil revela diferenciação ao nível de família (Mammalia: Chiroptera)
}

\author{
Sabrina Marchioro' ${ }^{1 *}$ \& João Marcelo Deliberador Miranda ${ }^{1}$ \\ 'Universidade Estadual do Centro Oeste, Guarapuava, Paraná, Brasil. E-mails: *sahmarchioro@gmail.com; \\ guaribajoao@yahoo.com.br.
}

\begin{abstract}
Trichological analysis of Brazilian bats reveals differentiation at family level (Mammalia: Chiroptera). The study of the microstructure of hair can be applied in several areas, such as: zoology, ecology, forensic sciences, archaeology, epidemiology and paleonthology, asides from being and useful and important tool on identifying mammal species. The objective of the present study was to analyze the patterns of cuticular microstructure of the hair of 67 bat species occurring in Brazil. The hairs were collected from the back of specimens deposited in a scientific collection (wet method). Later, these hairs were pressed in a glass slide for microscopy with a fine layer of uncolored nail polish for marking the cuticular impression, which was observed in optical microscope and compared to specific literature. The cuticular impressions were classified in eight morphological patterns: (1) narrow leaf shaped, (2) straight conoidal, (3) continuous straight conoidal, (4) ornamented wide conoidal, (5) uncontinuous ornamented conoidal, (6) cogged ornamented conoidal, (7) straight conoidal with bracts and (8) obliquous waved. The diferentiation was possible to the level of Family or Subfamily. By the cuticular morphology of hair, an identification key was made for bat's hairs to the level of Family or Subfamily (in the case of Phyllostomidae).
\end{abstract}

Keywords: identification, mastozoology, tricology.

Resumo. O estudo da microestrutura dos pelos pode ser aplicado em diversas áreas, como: zoologia, ecologia, ciências forenses, arqueologia, epidemiologia e paleontologia, além de ser uma ferramenta útil e importante na identificação das espécies de mamíferos. O objetivo do presente estudo foi analisar os padrões da microestrutura cuticular dos pelos de 67 espécies de morcegos ocorrentes no Brasil. Foram coletados pelos do dorso de espécimes depositados em coleção científica (via úmida). Posteriormente esses pelos foram prensados em lâminas para microscopia junto a uma fina camada de esmalte incolor, com o objetivo de marcar a impressão cuticular, que foi observada em microscópio óptico e comparada com literatura específica. As impressões cuticulares foram classificadas em oito padrões morfológicos: (1) folidáceo estreito, (2) conoidal liso, (3) conoidal contínuo liso, (4) conoidal ornamentado largo, (5) conoidal ornamentado descontínuo, (6) conoidal ornamentado dentado, (7) conoidal liso com bráctea e (8) ondeado oblíquo. Foi possível a diferenciação em nível de Família ou Subfamília. A partir da morfologia cuticular dos pelos foi construída uma chave para identificação de pelos de morcegos até o nível de família ou Subfamília (no caso de Phyllostomidae).

Palavras-chave: identificação, mastozoologia, tricologia. 


\section{INTRODUÇÃO}

Os mamíferos possuem anexos epidérmicos queratinizados exclusivos, conhecidos como pelos (AMmAN et al., 2002), que possuem uma complexa morfologia, podendo ser classificados em dois grupos principais: os subpelos ("underhair") são mais curtos, finos, ondulados e numerosos, e contribuem à termorregulação; e os pelos guarda ("overhair") são mais longos, grossos e menos numerosos, sobressaem-se na pelagem e contribuem principalmente à coloração desta, proteção mecânica e mecanorecepção (TEERINK, 2004). Quando se trata da morfologia interna, os pelos podem ser divididos em três camadas: medula, córtex e cutícula, da região mais interna para a mais externa, respectivamente (HAUSMAN, 1920).

A análise tricológica é uma ferramenta muito utilizada em estudos da microestrutura dos pelos, pois é útil na identificação de espécies de mamíferos, como por exemplo, felídeos, cervídeos, roedores, marsupiais e mustelídeos (MARTIN et al., 2009; VANSTREels et al., 2010; GOMEZ \& CASSINI, 2010; JOSHI et al., 2012; PORTELLA et al., 2013; SILVeIRA et al., 2013) e é aplicada em várias áreas, como zoologia, ecologia, ciências forenses, arqueologia, epidemiologia e paleontologia (QUADROS \& MONTEIRO-FILHO, 2006a).

Este tipo de técnica pode ser utilizada independente do pelo ser oriundo de animais vivos, ter passado por diferentes tratamentos como processo de decomposição, processo de digestão ou até mesmo processos de taxidermia, fixação em formaldeído e conservação em álcool em coleções científicas (QUADROS \& MONTEIROFILHO, 2006b; GOMES \& CASSINI, 2010; ROCHAMENDES, 2010; JOSHI et al., 2012; SILVEIRA et al., 2013), além disso, os pelos são mais úteis para identificação específica através da microestrutura de sua cutícula, que muitas vezes forma padrões morfológicos característicos para cada espécie de mamífero (CHERNOVA, 2002).

BENEDICT (1957) foi o pioneiro nos trabalhos tricológicos de morcegos ao desenvolver um guia de identificação tricológica, de várias regiões do mundo, revelando, que a maior parte das Famílias de morcegos não possui a parte medular do pelo (exceto: Pteropodidae, Rhinopomatidae e Megadermatidae; todas as três oriundas do Velho Mundo), diferentemente do restante dos outros mamíferos, onde os trabalhos cuticulares são acompanhados pelos medulares (QUADROS \& MONTEIRO-FILHO, 2006a e 2006b; MARTIN et al., 2009; GOMEZ \& CASSINI, 2010; SILVEIRA et al., 2013).

Dessa forma, embora tenham crescido os estudos tricológicos com outros mamíferos, em morcegos esse tipo de trabalho é mais desafiador, pois a pelagem é simplificada de duas formas, tanto pela falta da parte medular, quanto dos pelos guarda (BENEDICT, 1957). Este, talvez seja, um dos motivos para existirem poucos trabalhos nessa área, com quirópteros. Ainda há muitas suposições quanto a esse assunto, pois não se sabe ao certo o que acarretou a perda desses caracteres, que podem ter sido 
perdidos evolutivamente, já que ter pelos guarda e subpelos é uma condição derivada de todos os mamíferos (WeTterer et al, 2000; StRANEY, 1980). Assim, o objetivo do presente estudo foi descrever a morfologia dos pelos (pelos guarda e subpelos) de 67 espécies de morcegos ocorrentes no Brasil, além de também utilizarse da morfologia dos pelos para construir uma chave dicotômica detalhada baseada nos padrões tricológicos constituindo a primeira desse tipo para o país.

\section{MATERIAIS E MÉtodos}

Foi analisada a morfologia cuticular de pelos coletados de 67 espécies de morcegos ocorrentes no Brasil. Este total de espécies é pertencente a 37 gêneros e sete famílias. Os pelos foram coletados a partir de animais tombados na Coleção de Mastozoologia da Universidade Federal do Paraná (DZUP-UFPR), que estavam fixados em formaldeído (10\%) e conservados em álcool etílico (70\%), sendo amostrado um indivíduo de cada espécie (Tabela 1).

Tabela 1. Lista de espécies de morcegos amostrados distribuídos em sete famílias e 11 subfamílias com ocorrência no Brasil, totalizando 67 espécies (segundo o arranjo taxonômico e número de táxons proposto por NOGUEIRA et al., 2014).

\begin{tabular}{|c|c|c|c|}
\hline $\begin{array}{l}\text { Taxon (número total de espécies registradas no } \\
\text { Brasil) }\end{array}$ & $\begin{array}{l}\text { Número de } \\
\text { amostras por } \\
\text { taxa }\end{array}$ & $\begin{array}{l}\text { Representatividade } \\
(\%)\end{array}$ & $\begin{array}{c}\text { Número } \\
\text { tombo } \\
\text { (DZUP) }\end{array}$ \\
\hline Emballonuridae (17 espécies) & 1 espécie & $5.9 \%$ & - \\
\hline Rhynchonycteris naso (Wied-Neuwied, 1820) & - & - & 1910 \\
\hline Phyllostomidae (92 espécies) & 39 espécies & $42.4 \%$ & - \\
\hline Micronycterinae (1 de 9 espécies) & 1 espécie & $11.1 \%$ & \\
\hline Micronycteris megalotis (Gray, 1842) & - & - & 1675 \\
\hline Desmodontinae (3 espécies) & 2 espécies & $66.7 \%$ & - \\
\hline Desmodus rotundus (E. Geoffroy, 1810) & - & - & 2001 \\
\hline Diphylla ecaudata Spix, 1823 & - & - & 1319 \\
\hline Phyllostominae (17 espécies) & 11 espécies & $64.7 \%$ & - \\
\hline Chrotopterus auritus (Peters, 1856) & - & - & 1217 \\
\hline Lophostoma silvicolum d'Orbigny, 1836 & - & - & 2004 \\
\hline Lophostoma brasiliense Peters, 1866 & - & - & 2008 \\
\hline Macrophyllum macrophyllum (Schinz, 1821) & - & - & 1327 \\
\hline Mimom bennettii (Gray, 1838) & - & - & 1737 \\
\hline Mimom crenulatum (E. Geoffroy, 1803) & - & - & 1071 \\
\hline Phylloderma stenops Peters, 1865 & - & - & 1563 \\
\hline
\end{tabular}


Tabela 1. Continuação.

Phyllostomus hastatus (Pallas, 1767)

Phyllostomus discolor Wagner, 1843

Tonatia bidens (Spix, 1823)

Trachops cirrhosus (Spix, 1823)

Glossophaginae (9 espécies)

Anoura caudifer (E. Geoffroy, 1818)

Anoura geoffroyi Gray, 1838

Glossophaga soricina (Pallas, 1766)

Lonchophyllinae (7 espécies)

Lonchophylla thomasi J. A. Allen, 1904

$$
\text { Carolliinae (3 espécies) }
$$

Carollia perspicillata (Linnaeus, 1758)

“Glyphonycterinae” (5 espécies)

Trinycteris nicefori (Sanborn, 1949)

“Rhinophyllinae" ( 2 espécies)

Rhinophylla pumilio Peters, 1865

Stenodermatinae (34 espécies)

Artibeus fimbriatus Gray, 1838

Artibeus lituratus (Olfers, 1818)

Artibeus planirostris (Spix, 1823)

Artibeus concolor Peters, 1865

Artibeus cinereus (Gervais, 1856)

Artibeus obscurus (Schinz, 1821)

Artibeus gnomus Handley, 1987

Chiroderma villosum Peters, 1860

Chiroderma doriae Thomas, 1891

Platyrrhinus recifinus (Thomas, 1901)

Platyrrhinus incarum (Thomas, 1912)

Platyrrhinus lineatus (E. Geoffroy, 1810)
2049

2003

1395

811

3 espécies

$33.3 \%$

1021

1246

1020

1 espécie

$14.3 \%$

2039

1 espécie

$33.3 \%$

1907

1 espécie

$20 \%$

1440

1 espécie

$50 \%$

1103

18 espécies

$51.4 \%$

1004

778

2011

458

527

1086

1572

829

1406

879

2052

2009 
Tabela 1. Continuação.

Pygoderma bilabiatum (Wagner, 1843)

Sturnira lilium (E. Geoffroy, 1810)

Sturnira tildae de la Torre, 1959

Uroderma bilobatum Peters, 1866

Vampyressa pusila Wagner, 1843

Mormoopidae (3 espécies)

Pteronotus parnellii (Gray, 1843)

Noctilionidae ( 2 espécies)

Noctilio leporinus (Linnaeus, 1758)

Noctilio albiventris Desmarest, 1818

Thyropteridae (7 espécies)

Thyroptera tricolor Spix, 1823

\section{Molossidae (29 espécies)}

Molossus molossus (Pallas, 1766)

Molossus rufus (E. Geoffroy, 1805)

Molossops temminckii (Burmeister, 1854)

Molossops neglectus Williams \& Genoways, 1980

Nyctinomops laticaudatus (E. Geoffroy, 1805)

Eumops bonariensis (Peters,1874)

Eumops perotis (Schinz, 1821)

Tadarida brasiliensis (I. Geoffroy, 1824)

Vespertilionidae (28 espécies)

Myotinae (9 espécies)

Myotis nigricans (Schinz, 1821)

Myotis riparus Handley, 1960

Myotis albescens (E. Geoffroy, 1806)

Myotis dinellii Thomas, 1902

Myotis levis (I. Geoffroy, 1824)

Vespertilioninae (19 espécies)
1 espécie<smiles>C1CCCCC1</smiles>

2 espécies

$-$

$-$

1 espécie

$14.3 \%$

8 espécies

$27.6 \%$

-
-
-
-
-
-

14 espécies

5 espécies

$50 \%$

$55.5 \%$

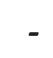

1082

1716

574

867

1230

1848

1339

1240

1106

1813

1419

596

1013

883

1369

963

2006

503

1371

526

1205

1380

82
16
4
7
30

9 espécies

$47.3 \%$ 
Tabela 1. Continuação.

\begin{tabular}{|c|c|c|c|}
\hline Lasiurus blossevillii (Lesson \& Garnot, 1826) & - & - & 1943 \\
\hline Lasiurus ega (Gervais, 1856) & - & - & 1333 \\
\hline Lasiurus cinereus (Beauvois, 1796) & - & - & 659 \\
\hline Eptesicus furinalis (d'Orbigny, 1847) & - & - & 1856 \\
\hline Eptesicus taddeii Miranda, Bernardi \& Passos, 2006 & - & - & 1009 \\
\hline Eptesicus diminutus Osgood, 1915 & - & - & 2005 \\
\hline Eptesicus brasiliensis (Desmarest, 1819) & - & - & 1781 \\
\hline Histiotus velatus (I. Geoffroy, 1824) & - & - & 1941 \\
\hline Histiotus montanus (Philippi \& Lanbeck, 1861) & - & - & 215 \\
\hline
\end{tabular}

As técnicas para retirada de pelos e preparação das lâminas foram de acordo com QuAdros \& MonTEIRO-FILHo (2006a). Tufos de pelos da região interescapular dos espécimes foram retirados com os dedos, preservando desde o bulbo até e ápice do mesmo. Logo após, estes foram prensados entre lâminas de microscopia, entre uma fina camada de esmalte, durante 15 minutos. Depois, estes pelos ficaram acondicionados em envelopes de papel, identificados com o nome da espécie (QUADROS \& Monteiro-Filho, 2006a). As impressões dos padrões cuticulares foram observadas através de microscópio óptico (Primo Star Zeiss ${ }^{\mathrm{TM}}$ ), no aumento de 400x. Após, foram fotografadas com uma câmera de cinco megapixels, acoplada ao microscópio, no mesmo aumento. Estas fotos ficaram armazenadas em arquivo digital (extensão .TIFF), a partir dos quais foram feitas as montagens das pranchas. Os padrões cuticulares foram classificados baseado em QuAdRos \& MONTEIRO-FILHO (2006b), com adaptações.

\section{RESULTADOS}

Asfamílias com maior representatividade de suas espécies, registradas no Brasil, no presente estudo foram Noctilionidae (100\%), seguida por Vespertilionidae (50\%) e Phyllostomidae $(42,4 \%)$. As Famílias Emballonuridae (5,9\%) e Thyropteridae $(14,3 \%)$ obtiveram as menores representatividades (Tabela 1), enquanto as famílias Furipteridae e Natalidae e a subfamília Lochorrhininae (Phyllostomidae) não foram amostradas.

Entre as famílias analisadas houve clara diferenciação na morfologia cuticular, em relação à dimensão e ornamentação das escamas, apresentando padrões imbricados (conoidal e folidáceo) e pavimentoso (ondeado). Foi verificado um padrão dentro de cada família, sendo que todas as espécies de cada família (ou subfamília dentro de Phyllostomidae) apresentaram o mesmo tipo morfológico na estrutura cuticular de seus pelos (Figuras 1-4). 

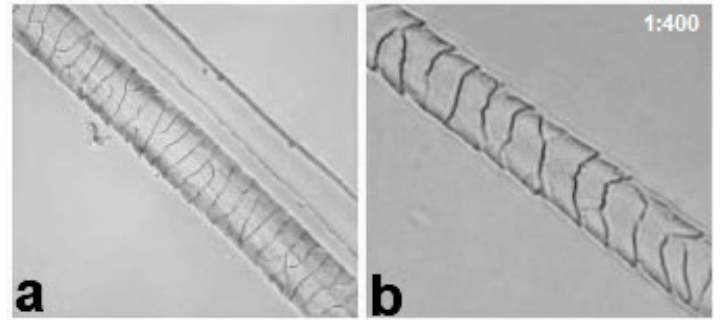

Figura 1. Impressões cuticulares de pelos guarda de Família Phyllostomidae: (a-b) Subfamília Desmodontinae (padrão ondeado oblíquo). Nas espécies (a) Desmodus rotindus (b) Diphylla ecaudata não há ângulos definidos na forma das escamas, e estas estão dispostas em diagonal com o eixo longitudinal do pelo, tornando-os diferentes das demais espécies da Família.
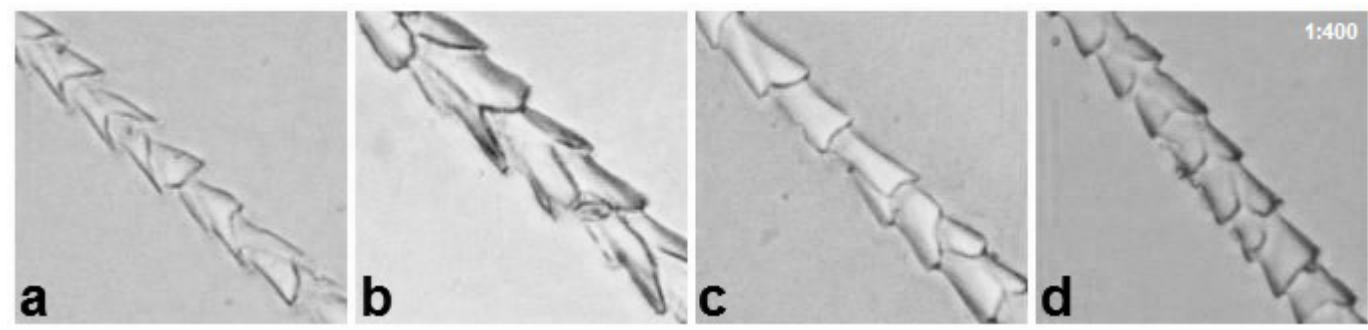

Figura 2. Impressões cuticulares de pelos guarda de Família Vespertilionidae (padrão folidáceo estreito): (a) Eptesicus furinalis; (b) Lasiurus cinereus; (c) Eptesicus brasiliensis e (d) Histiotus velatus.
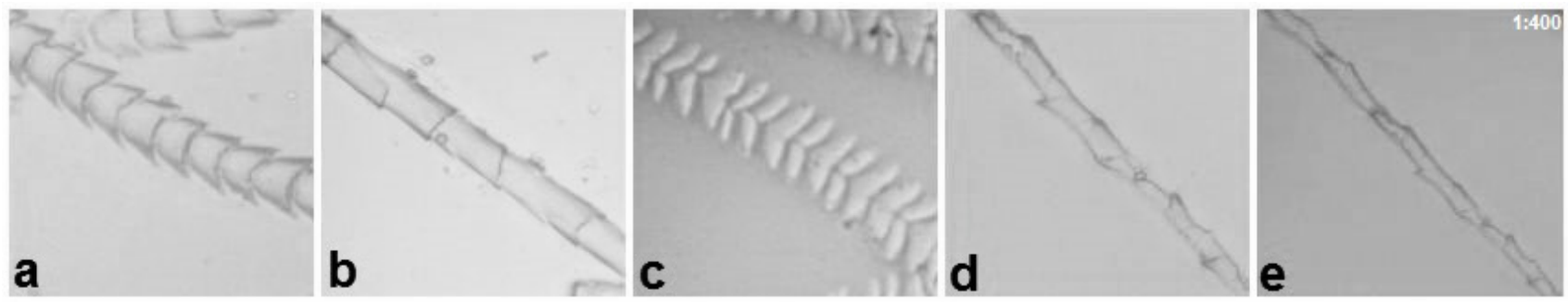

Figura 3. Impressões cuticulares de pelos guarda de: (a) Thyroptera tricolor - Família Thyropteridae (padrão conoidal liso); (b) Pteronotus parnellii - Família Mormoopidae (padrão conoidal contínuo liso); (c) Rhynchonycteris naso - Família Emballonuridae (padrão conoidal ornamentado largo); (d) Noctilio leporinus e (e) Noctilio albiventris -Família Noctilionidae (padrão conoidal ornamentado descontínuo).
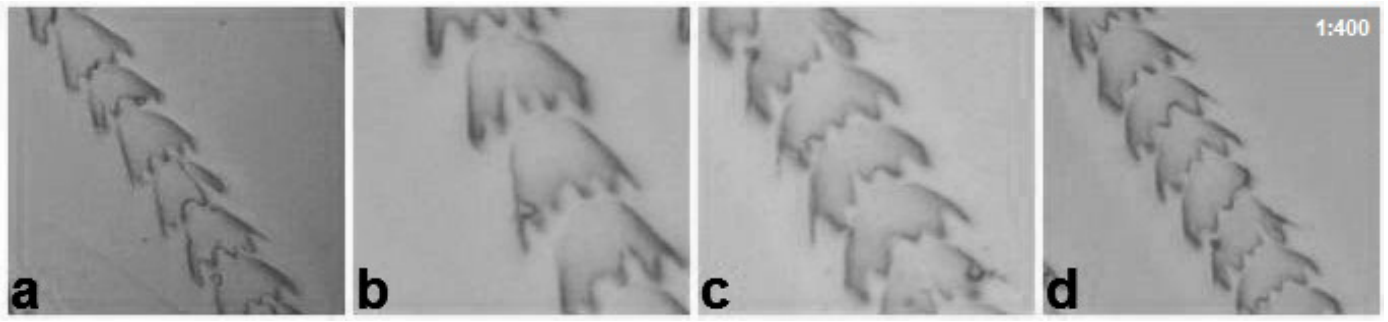

Figura 4. Impressões cuticulares de pelos guarda de Família Molossidae (padrão conoidal ornamentado dentado): (a) Molossus molossus; (b) Eummops bonariensis; (c) Nyctinomops laticaudatus e (d) Tadarida brasiliensis. 
A variação morfológica cuticular das Glossophaginae, Lonchophyllinae, Caroliinae, 67 espécies amostradas foram classificadas em "Glyphonycterinae", Rhinophyllinae e oito padrões morfológicos: (1) ondeado oblíquo Stenodermatinae) (Figura 5a-f).

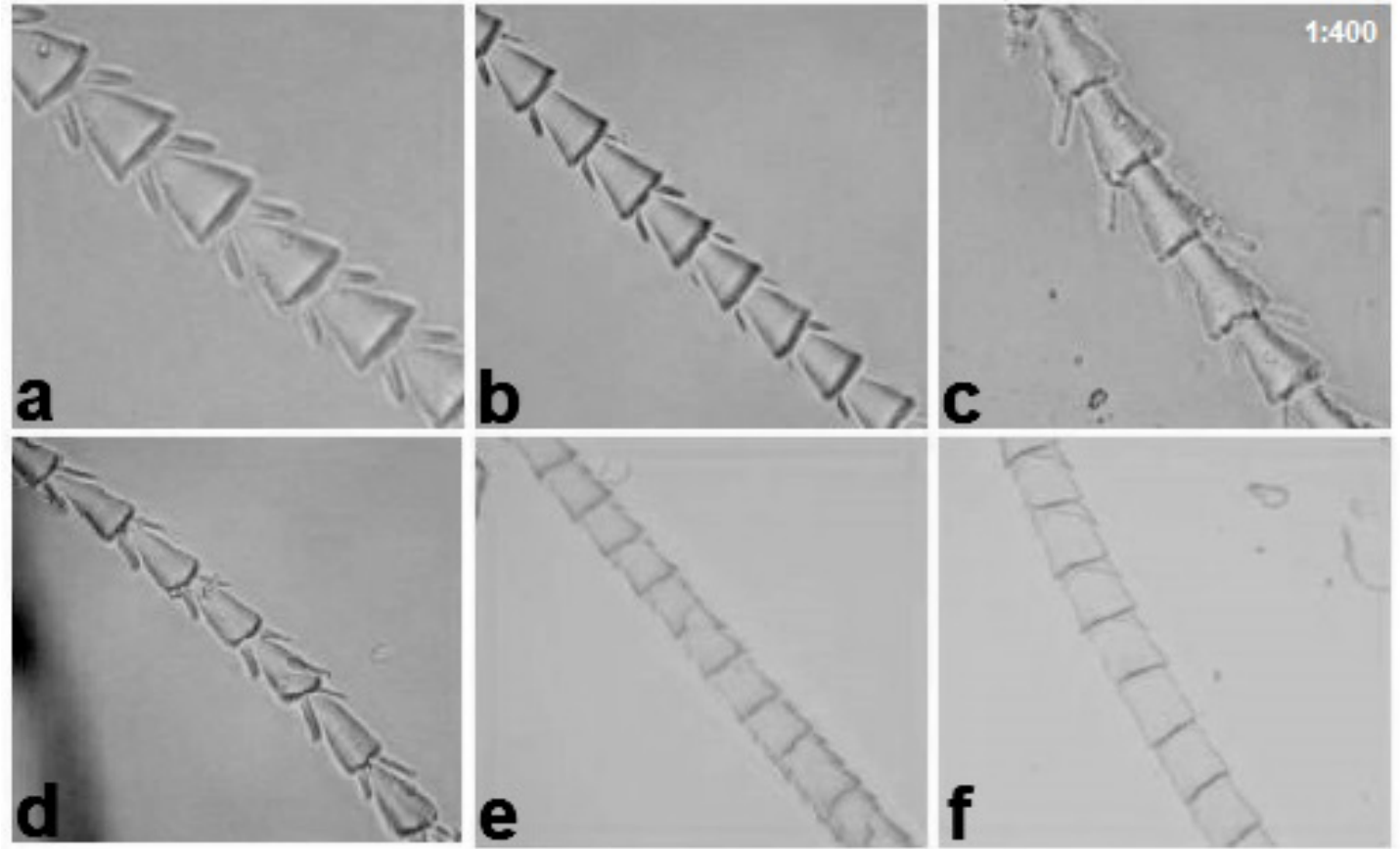

Figura 5. Impressões cuticulares de pelos guarda de Família Phyllostomidae: (a-f) Subfamílias: Micronycterinae, Phyllostominae, Glossophaginae, Lonchophyllinae, Caroliinae, "Glyphonycterinae", Rhinophyllinae e Stenodermatinae (padrão conoidal liso com bráctea). Nas espécies (c) Carollia perspicillata e (d) Rhinophylla pumilio as "brácteas" são mais longas e abertas, ou destacadas do eixo do pelo, diferenciando-as das demais espécies analisadas da Família. Nas espécies (e) Mimon bennetti e (f) Mimon crenulatum os cones são mais unidos, e com as "brácteas" laterais menores que as demais espécies analisadas da Família.

(Phyllostomidae - Desmodontinae) (Figura 1a-
b); (2) folidáceo estreito (Vespertilionidae)

(Figura 2); (3) conoidal liso (Thyropteridae)

(Figura 3a); (4) conoidal contínuo liso (Mormoopidae) (Figura 3b); (5) conoidal ornamentado largo (Emballonuridae) (Figura

3c); (6) conoidal ornamentado descontínuo (Noctilionidae) (Figura 3d-e); (7) conoidal ornamentado dentado (Molossidae) (Figura 4) e (8) conoidal liso com bráctea (Phyllostomidae

- Subfamílias: Micronycterinae, Phyllostominae,
Na Família Phyllostomidae (Figuras 1 e 5) o padrão cuticular das escamas, foi classificado com base nas descrições propostas por QUADROS \& Monteiro-Filho (2006b), como acréscimo da descrição da ornamentação em forma de "bráctea". O padrão observado é o conoidal liso, e na borda das escamas aparece uma ornamentação em forma de "bráctea" que parte da base do cone, como uma pequena folha. Dentro desta mesma Família houve variações. Nas espécies analisadas das Subfamílias Carolliinae e "Rhinophyllinae" 
(Carollia perspicillata e Rhinophylla pumilio), as "brácteas" dos cones do padrão conoidal são mais longas e mais abertas, ou destacadas do eixo do pelo, diferenciando-as dos demais (Figura $5 c-d$ ). As duas espécies do Gênero Mimon (Subfamília Phyllostominae), apresentaram também uma pequena divergência, onde seus cones são mais unidos e com as "brácteas" laterais menores que as demais espécies de Phyllostomidae (Figuras 5e-f). Estas modificações, apesar de pequenas, podem ser úteis como caractere diagnóstico. As duas espécies da Subfamília Desmodontinae (Desmodus rotundus e Diphylla ecaudata) foram as mais diferentes dentre as espécies da Família
Phyllostomidae (Figura 1a-b), onde verificou-se o padrão cuticular ondeado oblíquo e a forma das escamas não apresenta ângulos definidos e estas estão dispostas em diagonal com o eixo longitudinal do pelo.

A morfologia diferencial dos padrões tricológicos permite a identificação de pelos morcegos ao nível de família (e a subfamília em Desmodontinae, dentro de Phyllostomidae). Sendo apresentada abaixo uma chave dicotômica de identificação de pelos de morcegos e que pode ser uma ferramenta utilizada em trabalho de dieta de felídeos e corujas, bem como inventários em abrigos desocupados de morcegos.

\section{Chave dicotômica para identificação de famílias de morcegos brasileiros através do padrão cuticular de pelos}

1. Bordas das escamas pavimentosas, forma ondeada e orientação oblíqua.

Phyllostomidae:

\section{Desmodontinae (Fig. 1a-b)}

1'. Bordas das escamas imbricadas, forma folidácea ou conoidal.

2. Forma folidácea e dimensão estreita

2'. Forma conoidal.

3. Bordas das escamas lisas......

3'. Bordas das escamas ornamentadas.

4. Escamas de comprimento similar à largura (intermediárias). Thyropteridae (Fig. 3a)

4'. Escamas de comprimento maior que a largura (estreitas). Mormoopidae (Fig. 3b)

5. Escamas de comprimento menor que a largura (largas). Emballonuridae (Fig. 3c)

5'. Escamas de comprimento similar (intermediárias) ou maior (estreitas) que a largura.....

6. Escamas de comprimento maior que a largura (estreitas) e de bordas descontínuas. 
6'. Escamas de comprimento similar à largura (intermediárias)

7. Escamas de bordas ornamentadas e dentadas Molossidae (Fig. 4)

7’. Escamas de bordas lisas e providas de uma "bráctea" Phyllostomidae (demais Subfamílias) (Fig. 5a-f)

\section{DIscussão}

A análise tricológica funciona bem na identificação específica de pequenos mamíferos Neotropicais não voadores (marsupiais e roedores) (ABREU et al., 2001) e pode ser usada na discriminação de espécies crípticas (SILVEIRA et al., 2013). Esta técnica também funciona em inventários de alguns tipos de mamíferos carnívoros, como mustelídeos (GOMEZ \& CASSINI, 2010) e de felídeos (PORTELLA et al., 2013).

Os táxons de morcegos analisados no presente estudo representaram $77,7 \%$ das famílias; $54,4 \%$ dos gêneros e 38,2\% das espécies ocorrentes no Brasil (NogueIRA et al., 2014). Os padrões cuticulares de escamas pavimentosas e imbricadas encontrados corroboram com BENEDICT (1957) e QuAdRos \& MONTEIRO-FILHO (2006a), porém foram encontradas estruturas novas, como a ornamentação do tipo "bráctea" nas escamas dos pelos dos Phyllostomidae (nãoDesmodontinae).

BENEDICT (1957) afirma que na Família Phyllostomidae cada escala cuticular circunda o eixo do pelo inteiro, e essas escalas sucessivas divergem mais no lado oposto do filamento, fazendo com que cada escala apareça em forma de "V", porém isto não ocorre nos desmodontíneos, pois suas escalas são completamente pressionadas no filamento, sugerindo que isto deve ser uma condição primitiva para esta Família. Sendo assim, esta Subfamília realmente precisa de estudos mais aprofundados.

VANSTREELS e colaboradores (2010) e RINALDI e colaboradores (2015) encontraram dificuldades na identificação de felídeos, através da tricologia, pois os pelos tinham muitas semelhanças entre si ou ocorria sobreposição de espécies, mostrando que a microestrutura de seus pelos é desafiadora e requer um exame cuidadoso de caracteres sutis e/ou ser limitada principalmente às aplicações que não requerem identificação específica, apenas em níveis taxonômicos superiores, como o que ocorreu no presente trabalho.

Abordagens morfométricas também podem ser de grande valia quando associadas a este método, como GoMEZ \& CASSINI (2010) nos mostram em seu estudo na identificação de mustelídeos na Patagônia. Também foram utilizadas análises estatísticas e padrões de coloração, que os ajudaram a chegar aos níveis de gênero e em alguns casos, de espécie, durante a identificação.

Neste trabalho, a identificação da Ordem Chiroptera, foi possível até o nível de Famílias ou Subfamílias, sendo imprecisa a 
diferenciação de gêneros ou espécies. Mas ainda assim, foi possível a construção de uma chave de identificação, que pode ter grande utilidade para pesquisadores que estudam, por exemplo, dieta de animais silvestres, pois caso seja encontrado pelos nas fezes, é possível ter uma ideia se são pelos de quirópteros, e a qual Família pertence.

Através da microestrutura dos pelos guarda, como suas diferenças interespecíficas e dimensões de tamanho, podemos utilizálos como uma ferramenta suplementar de identificação de morcegos, principalmente através de microscopia eletrônica e de chaves de identificação, como foi feito para Molossídeos do Novo Mundo (STAADEN \& Jones, 1997), para morcegos do Colorado (EUA) (AMMAN et al., 2002) em morcegos de Porto Rico (SCHAETZ et al., 2009).

Sendo assim, novos estudos, como de micrografia eletrônica, espectofotometria, cortes histológicos da pele, uso de ferramentas moleculares, de análises morfométricas em conjunto com análises estatísticas, desses e de outras espécies de morcegos que não foram utilizadas neste trabalho, devem ser realizados afim de buscar novos padrões da microestrutura dos pelos guarda, e aprofundar os conhecimentos sobre a tricologia dos morcegos brasileiros.

\section{AgRADECIMENTOS}

AoConselho Nacional de Desenvolvimento Científico e Tecnológico, CNPq, pela concessão da bolsa de Iniciação Científica (processo número 129977/2013-6); ao Prof. Dr. Fernando de
Camargo Passos pelo acesso à Coleção Científica de Mastozoologia da Universidade Federal do Paraná.

\section{REFERÊNCIAS BIBLIOGRÁFICAS}

Abreu, M.S.L.; Christoff, A.U. \& Vieira, E.M. 2001. Identificação de marsupiais do Rio Grande do Sul através da microestrutura dos pelos guarda. Biota Neotropica 11(3): 391-400.

AMmAN, B.R..; OWEN, R.D. \& BRADLEY, R.D. 2002. Utility of hair structure for taxonomic discrimination in bats, with an example from the bats of Colorado. Occasinal Papers, Museum of Texas Tech University 216: 1-15.

BENEDICT, F.A. 1957. Hair structure as a generic character in bats. University of California Publications in Zoology 59: 285-548.

CHERnOVA, O.F. 2002. Architectonic and diagnostic significance of hair cuticle. Biology Bulletin 29 (3):238-247.

GoMEZ, J.J. \& CASSINI, M.H. 2010. Uso de pelos de guardia para la identificación de mustélidos costeros en la Patagonia.

Revista de Biología Marina y Oceanografía 45(2):365-370.

HAUSMAN, L.A. 1920. Structural characteristics of the hair mammals. American Naturalist 54(635):496-523.

JOSHI, H.R.; GAIKWAD, S.A.; TOMAR, M.P.S. \& SHRIVASTAVA, K. 2012. Comparative 
Trichology of Common Wild Herbivores of India. Pelagia Research Library 3(6):34553458.

Martin, P.S.; Gheler-Costa, C. \& Verdade, L.M. 2009. Microestruturas de pelos de pequenos mamíferos não-voadores: chave para identificação de espécies de agrossistemas do estado de São Paulo, Brasil. Biota Neotropica 9(1): 233-241.

Nogueira, M.R.; LiMA, I.P.; MORATELLI, R.; TAVARES, V.C.; Gregorin, R. \& Peracchi, A. 2014. Checklist of Brazilian bats, with comments on original records. CheckList: Journal of species lists and distribution 10(4): 808821.

PORTELLA, T.P.; BILSKI, D.R.; PASSOS, F.C. \& PIE, M.R. 2013. Assessing the efficacy of hair snares as a method for noninvasive sampling of Neotropical felids. Zoologia 30(1):49-54.

Quadros, J. \& Monteiro-Filho, E.L.A. 2006a. Coleta e preparação de pelos de mamíferos para identificação em microscopia ótica. Zoologia 23(1):274-278.

Quadros, J. \& MonteIRo-Filho, E.L.A. 2006b. Revisão conceitual, padrões microestruturais e proposta nomenclatória para os pelos-guarda de mamíferos brasileiros. Zoologia 23(1):279-292.

Rinaldi, A.R.; Rodriguez, F.H. \& PASSOS, F.C. 2015. Is it possible to identify four small Neotropical felids (Carnivora: Felidae) based on hair microstructure? Zoologia

$$
\text { 32(1): 77-82. }
$$

Rocha-Mendes, F.; MiKICH, S.B.; QuAdroS, J. \& Pedro, W.A. 2010. Feeding ecology of carnivores (Mammalia, Carnivora) in Atlantic Forest remnants, Southern Brazil. Biota Neotropica 10(4): 21-30.

SCHAETZ, B.A.; KURTA, A.; ROdRÍGUEZ-DSURÁN, A.; Münzer, O.M. \& FOster, R. 2009. Identification of bats in Puerto Rico using the scanning eléctron microscope to examine body hairs. Caribbean Journal of Science 45(1):125-130.

Silveira, F.; Sbalqueiro, I.J. \& Monteiro-Filho, E.L.A. 2013. Identificação das espécies brasileiras de Akodon (Rodentia: Cricetidae: Sigmodontinae) através da microestrutura dos pelos. Biota Neotropica 13(1): 338345.

StAaden, M.J. VAN \& JONes, J.K. JR. 1997. Comparative morphology of dorsal hair of New World bats of the family Molossidae. In: Arroyo-Cabrales, J. \& Polaco, O. J. eds. Homenaje al Profesor Ticul Álvarez. Mexico, Instituto Nacional de Antropología e Historia. p.373-391.

STRANEY, D.O. 1980. Relationships of phyllostomatine bats: evaluation of phylogenetic hypotheses. Ph.D. dissertation. University of California, Berkeley.

TeERINK, B.J. 2004. Hair of West European mammals: atlas and identification key. 
Cambridge University Press, Cambridge.

VANstreels, R.E.T.; Ramalho, F.P. \& AdaniA, C.H. 2010. Microestrutura de pelos guarda de felídeos brasileiros: considerações para a identificação de espécies. Biota Neotropica 10 (1): 332-337.

WetTerer, A.L.; ROCKMAN, M.V. \& SIMMONS, N.B. 2000. Phylogeny of phyllostomid bats: data from diverse morphological systems, sex chromosomes, and restricted sites. Bulletin of the American Museum of Natural History 248: 46-67.

Recebido: 19/05/2017

Revisado: 02/07/2017

Aceito: 07/07/2017 